\title{
RELAÇÕES DE CAUSA E EFEITO EM TESTE DE PERFORMANCE DE BOBINAS DE IGNIÇÃO
}

\author{
Henrique Silva $^{1}$, Ruan Carvalho Mayworm ${ }^{1,2}$ \\ ${ }^{1}$ Robert Bosch Ltda - Gasoline Systems - Engineering Testing Center - Latin America \\ (GS/ETC-LA), Campinas, SP, Brazil. \\ ${ }^{2}$ Programa INOVA Talentos - MCTIC, CNPq e IEL \\ E-mails: henrique.silva2@br.bosch.com, rcmayworm@gmail.com
}

\section{RESUMO}

A bobina de ignição tem a função de gerar uma alta tensão com energia suficiente para provocar centelhas entre os eletrodos da vela de ignição, cujo objetivo é iniciar o processo de combustão em uma máquina de combustão interna. Para verificar a performance da bobina de ignição são executados dois testes funcionais, o teste de oferta de tensão e o teste de energia da faísca. No primeiro, verifica-se a tensão enviada à carga $\left(\mathrm{U}_{2}\right)$, a corrente no enrolamento primário $\left(\mathrm{I}_{1}\right)$ e o tempo de descida/subida da centelha $\left(\mathrm{t}_{\mathrm{r}}\right)$, no segundo, verifica-se a energia da faísca $\left(\mathrm{W}_{\mathrm{sp}}\right)$, a corrente no enrolamento secundário $\left(\mathrm{I}_{\mathrm{sp}}\right)$ e a duração da faísca $\left(t_{\mathrm{sp}}\right)$. Os resultados obtidos são a base para o desenvolvimento de novos produtos, bem como para a manutenção de um produto de série no mercado. Logo, é notória a grande importância que os ensaios de performance tem no processo de testes de confiabilidade do produto. Portanto, neste trabalho, foi realizado um estudo que permite um melhor entendimento desses ensaios, abordando a influência da variação dos parâmetros de entrada nos parâmetros de saída da bobina de ignição. Portanto, para uma determinada faixa de valores, mapeou-se qual o comportamento da bobina de ignição quando alterados parâmetros como: tensão de bateria, carga da bobina de ignição e tempo de carregamento do enrolamento primário, ou seja, verificou-se a resposta do produto medindo-se os parâmetros relevantes para os ensaios de oferta de tensão e de energia da centelha. Como resultado do estudo, tem-se as equações que regem as relações de causa e efeito dos parâmetros em análise, essas equações foram determinadas de forma experimental, utilizando dados de testes e a ferramenta de regressão do Microsoft Excel®. Concluiu-se que, com os dados gerados, o laboratório tem uma ferramenta adicional para justificar alterações em seus setups e identificar fenômenos ocorridos durante as medições, tais como o ponto de saturação magnética e o ponto de saturação elétrica das amostras. Ademais, por meio das equações de causa e efeito, coeficientes de sensibilidade podem ser obtidos para futuras estimativas de incerteza de medição.

\section{INTRODUÇÃO}

A energia resultante de um arco elétrico (centelha), gerado entre os eletrodos de uma vela de ignição, é a fonte externa de energia necessária para realizar a queima da mistura ar- 
combustível de um motor de combustão interna. A bobina de ignição, vela de ignição, cabos de ignição e o estágio de potência, formam o sistema de ignição. Ele é responsável por gerar os arcos elétricos, os quais fornecem a energia suficiente para que a queima da mistura de ar e combustível seja adequada, além de garantir que a centelha aconteça no momento exato do ciclo do motor. [1,2]

Dentre os componentes do sistema de ignição, a bobina de ignição tem a função de gerar a alta tensão requerida para provocar centelhas na vela de ignição, cujo objetivo é iniciar o processo de combustão da mistura ar-combustível. [3]

As regulamentações sobre emissões ambientais veem impondo limites à poluição por motores de combustão interna. Os maiores contribuintes para os drásticos aumentos nas emissões são a falta de ignição e a combustão incompleta. Por tanto, a fim de mitigar tais fatores, o projeto da bobina vem sendo continuamente melhorado, para satisfazer as crescentes demandas impostas pelo motor a gasolina e/ou etanol. Adicionalmente à questão das emissões ambientais, existe uma tendência no aumento da severidade em relação às medições veiculares para os níveis de emissões eletromagnéticas, principalmente para emissões irradiadas, onde os componentes do sistema de ignição têm um peso significativo. [1,2]

Devido a todas estas exigências impostas ao setor automotivo, as características de diversos produtos, tal como a bobina de ignição, necessitam ser controladas. Como exemplos de ensaios realizados em bobinas de ignição, podem-se citar o ensaio funcional de oferta de tensão, no qual verifica a tensão enviada à carga $\left(\mathrm{U}_{2}\right)$, a corrente gerada no enrolamento primário da bobina $\left(\mathrm{I}_{1}\right)$ e o tempo de descida/subida da centelha, denominado rise time ( $\mathrm{t}_{\mathrm{r}}$ ), e o ensaio funcional de energia da faísca, no qual verifica-se a energia da faísca $\left(\mathrm{W}_{\mathrm{sp}}\right)$, a duração da faísca $\left(\mathrm{t}_{\mathrm{sp}}\right)$ e a corrente no enrolamento secundário $\left(\mathrm{I}_{\mathrm{sp}}\right)$. Os resultados obtidos nestes ensaios servem de base para o desenvolvimento de novos produtos, assim como para a manutenção ou inclusão de um produto de série no mercado.

De um modo geral, as montadoras de automóveis adquirem peças (bombas de combustível, bobina de ignição e etc.) levando em consideração, além de outros aspectos, os resultados obtidos nos testes de validação, onde a verificação funcional tem papel fundamental, pois possibilita averiguar os parâmetros que irão demonstrar se a amostra está aprovada ou reprovada. Logo, é necessário ter o domínio das relações entre os parâmetros envolvidos nesses ensaios, assim é possível realizar testes de qualidade, evitando-se pseudo-falhas e auxiliando a tomada de decisões.

O estudo de causa e efeito nos ensaios funcionais tem papel fundamental para o pleno entendimento das variações produzidas nos resultados, em função das variações nas grandezas de entrada. Dessa maneira, diante de um resultado duvidoso, é possível identificar se a variação ocorre devido a uma variação natural do sistema ou se, realmente, é um desvio.

Sendo assim, é necessário conhecer os efeitos causados por tais variações, para tal, as principais grandezas de entrada foram variadas: tensão de bateria (T), carga do secundário (F), tempo de carregamento do primário, denominado dwell time $\left(\mathrm{t}_{\mathrm{dwell}}\right)$, e tensão Zener $\left(\mathrm{U}_{\mathrm{z}}\right)$. A partir disso, realizaram-se medições visando compreender os impactos do incremento ou decremento dos valores de entrada sobre as grandezas de saída, sendo elas: tensão enviada à carga $\left(\mathrm{U}_{2}\right)$, a corrente gerada no enrolamento primário da bobina $\left(\mathrm{I}_{1}\right), \mathrm{o}$ 
tempo de descida/subida da centelha, denominado rise time $\left(\mathrm{t}_{\mathrm{r}}\right)$, energia da faísca $\left(\mathrm{W}_{\mathrm{sp}}\right)$, duração da faísca $\left(t_{\mathrm{sp}}\right)$ e a corrente no enrolamento secundário $\left(\mathrm{I}_{\mathrm{sp}}\right)$.

Desta forma, os laboratórios que realizam ensaios em bobinas de ignição estarão em condições de identificar possíveis fontes de variação ocasionados pelas grandezas de entrada. Além disso, os coeficientes de sensibilidade, utilizados para expressar a incerteza de medição, podem ser calculados a partir das equações obtidas neste estudo. Portanto, este estudo servirá de base para o melhor entendimento dos ensaios de oferta e energia, além de servir como suporte para futuros cálculos de incerteza de medição.

\section{CONCEITOS TEÓRICOS BÁSICOS DE UM SISTEMA DE IGNIÇÃO}

Em um sistema de ignição, a bobina de ignição tem papel fundamental, basicamente, consiste de dois enrolamentos de cobre acoplados magneticamente, o enrolamento primário e o enrolamento secundário. A energia armazenada no enrolamento primário (entrada de baixa tensão), é transformada em um sinal de alta tensão, que é verificado no enrolamento secundário (saída do componente). O funcionamento da bobina de ignição baseia-se na lei da indução de Faraday, ou seja, a força eletromotriz induzida, é igual ao negativo da taxa de variação do fluxo magnético no tempo. A forma matemática da lei da indução de Faraday é mostrada na Equação 1. [4]

$$
\varepsilon=-\frac{\partial \phi_{\mathrm{B}}}{\partial \mathrm{t}}
$$

Na qual:

$\varepsilon=$ Força eletromotriz [V]

$\phi_{\mathrm{B}}=$ Fluxo magnético $[\mathrm{Wb}]$

Na Figura 1, encontra-se o esquema simplificado de um sistema de ignição, no qual demonstra como os componentes estão interconectados em função de gerar a energia necessária para que o evento de ruptura do dielétrico da vela de ignição ocorra.

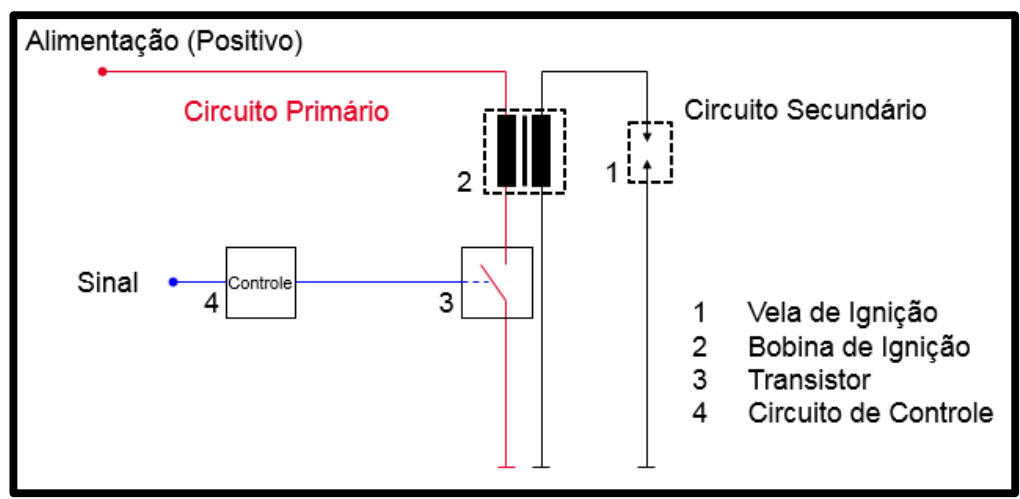

Figura 1. Esquema básico de um sistema de ignição. [5]

A bobina de ignição, representada no bloco 2 da Figura 1, possui dois principais parâmetros de entrada, que são a tensão proveniente da bateria e a corrente elétrica do primário. Na saída do componente, tem-se a tensão induzida, que deve conter energia suficiente para romper o dielétrico e produzir a faísca.

O sistema de controle eletrônico das bobinas de ignição, representado nos blocos 3 e 4 da Figura 1, pode ser interno à unidade de comando do motor ou embarcado junto ao 
componente. Esse controle determina quando o processo de ignição é iniciado e o momento em que ele é extinto. Isso é feito comutando-se um transistor, que quando acionado, permite o carregamento do enrolamento primário, então, é armazenada a energia suficiente para suprir a demanda do processo de combustão. Posteriormente, ocorre a extinção da corrente através do desligamento do transistor, o que permite uma grande variação de fluxo magnético no tempo, sendo assim, a indução de tensão no enrolamento secundário é viabilizada e a energia armazenada é descarregada na vela de ignição. O controle preciso da duração da faísca é essencial para garantir a performance do motor e um eficiente controle dos níveis de emissões. [1]

No bloco 1 da Figura 1, tem-se a vela de ignição, que através de um condutor isolado do motor, transmite a alta tensão para um eletrodo conectado à massa, e então a centelha é gerada. A vela é montada de forma que seu eletrodo conectado à massa fique dentro da câmara de combustão. Com esse processo a mistura ar/combustível entra em ignição por intermédio da faísca elétrica que se forma entre os elétrodos.

Predominantemente são encontrados dois tipos de bobinas de ignição, a bobina 2x2, com duas terminações de saída (uma bobina para dois cilindros) e a bobina 1x1, com uma terminação de saída (uma bobina por cilindro). Na Figura 2, é possível observar o esquema de ligação de uma bobina tipo 1x1, modelo utilizado neste estudo. [5]

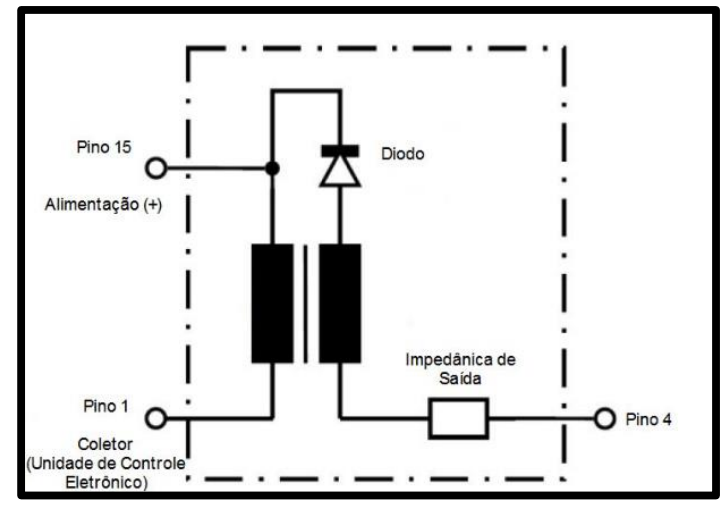

Figura 2. Esquema simplificado de uma bobina de ignição 1x1. [6]

O sistema apresentado na Figura 3, demonstra de maneira simplificada, o esquema de ligação da bobina $1 \times 1$, que nesse caso, possui o sistema de controle eletrônico dentro da central de comando do veículo, onde está armazenado o mapeamento do tempo de carregamento da bobina de ignição, comumente designado como dwell time (tempo em $O N$ ), permitindo o controle dos níveis de energia da faísca em diferentes rotações.

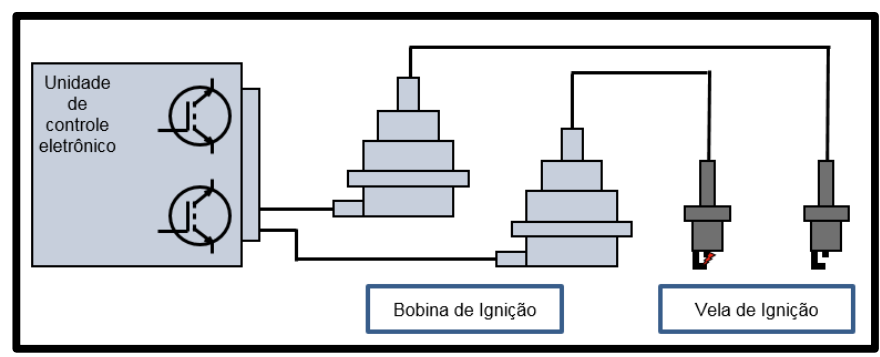

Figura 3. Circuito de controle da bobina de ignição tipo Single Ended Coil. [5] 
A energia armazenada no primário é determinada pela indutância e pela corrente do primário (no momento do desligamento). A Equação 2, relaciona esses parâmetros. [5]

$$
\mathrm{E}_{1}=\frac{1 \cdot \mathrm{L}_{1} \cdot \mathrm{I}_{1}^{2}}{2}
$$

Na qual:

$\mathrm{E}_{1}=$ Energia do primário $[\mathrm{J}]$

$\mathrm{L}_{1}=$ Indutância do primário $[\mathrm{H}]$

$\mathrm{I}_{1}=$ Corrente do primário $[\mathrm{A}]$

A energia da faísca pode ser controlada pela corrente do primário, que é descrita pela Equação 3. [5]

$$
I_{\text {prim }}(t)=\frac{U_{\text {batt }}-U_{c e}}{R_{\text {prim }}} \cdot\left[1-\exp \left(\frac{-R_{\text {prim }}}{L_{\text {prim }}} \cdot t\right)\right]
$$

Na qual:

$\mathrm{I}_{\text {prim }}=$ Corrente do primário $[\mathrm{A}]$

$\mathrm{U}_{\text {batt }}=$ Tensão de alimentação [V]

$\mathrm{U}_{\mathrm{ce}}=$ Queda de tensão do driver $[\mathrm{V}]$

$\mathrm{R}_{\text {prim }}=$ Resistência do primário $[\Omega]$

$\mathrm{L}_{\text {prim }}=$ Indutância do primário $[\mathrm{H}]$

$\mathrm{t}=$ Tempo $[\mathrm{s}]$

A variação do fluxo magnético, ocasionada pela variação da corrente do primário, induz a tensão no secundário, que pode ser descrita conforme Equação 4. [5]

$$
\mathrm{U}_{\text {sec }}(\mathrm{t})=\sqrt{\frac{\mathrm{L}_{\text {sec }}}{\mathrm{C}_{\text {sec }}}} \cdot \mathrm{I}_{\text {sec } \_\mathrm{i}_{\mathrm{i}}} \cdot \operatorname{sen}(\omega \cdot \mathrm{t})
$$

Na qual:

$\mathrm{U}_{\mathrm{sec}}=$ Tensão do secundário [V]

$\mathrm{L}_{\mathrm{sec}}=$ Indutância do secundário $[\mathrm{H}]$

$\mathrm{C}_{\mathrm{sec}}=$ Capacitância do secundário $[\mathrm{F}]$

$\mathrm{I}_{\mathrm{sec} \_\mathrm{i}}=$ Corrente inicial do secundário [A]

$\omega=$ Frequência angular $[\mathrm{rad} / \mathrm{s}]$

$\mathrm{t}=$ Tempo $[\mathrm{s}]$

A frequência angular pode ser descrita segundo a Equação 5. [5]

$$
\omega=\sqrt{\frac{1}{\mathrm{~L}_{\mathrm{sec}} \cdot \mathrm{C}_{\mathrm{sec}}}}
$$

Na qual:

$\mathrm{L}_{\mathrm{sec}}=$ Indutância do secundário $[\mathrm{H}]$

$\mathrm{C}_{\mathrm{sec}}=$ Capacitância do secundário $[\mathrm{F}]$

\section{MATERIAS E MÉTODOS}

O objetivo desse trabalho é apresentar as relações de causa e efeito das medições realizadas nos ensaios de oferta de tensão e energia da centelha, realizado nas bobinas de 
ignição. Para tal, identificou-se os principais parâmetros de entrada e saída do sistema, após, variou-se as grandezas de entrada e observou-se a sua influência nas grandezas de saída. Por fim, por meio de regressões lineares ou polinomiais, obtiveram-se equações que relacionam a sensibilidade entre as grandezas de entrada e as de saída. Mais detalhes são apresentados a seguir.

\subsection{Ensaio de Oferta de Tensão}

Os testes de confiabilidade são realizados para validações, requalificações e modificações, e devem ser selecionados de acordo com a necessidade de cada fim. Todas as sequências de testes de confiabilidade contêm, repetidas vezes, o controle de performance da bobina de ignição. A cada etapa de testes é necessário o controle funcional das amostras, para averiguar qual a condição das características da bobina, assim é possível avaliar se a amostra está robusta em relação à cada etapa da sequência de testes. O controle de parâmetros é feito levando em consideração os critérios de aprovação, ou seja, compara-se o valor retirado da medição em bancada com o valor da especificação.

O objetivo do ensaio de oferta de tensão é verificar a performance da bobina de ignição, analisando o nível de alta tensão no secundário e a corrente medida no primário. $\mathrm{O}$ procedimento consiste em aplicar um sinal de potência nos terminais de entrada do componente, verificar o nível de alta tensão entregue na saída, a velocidade com que o evento ocorre e analisar a corrente de pico medida na entrada do circuito. Esses parâmetros são avaliados conforme requisitos previamente estabelecidos e então uma decisão pode ser tomada levando-os em consideração.

O ensaio foi realizado na bancada de performance de bobina de ignição no laboratório da engenharia de testes. Os equipamentos de teste utilizados para a execução das medições foram: osciloscópio, gerador de funções, fonte de alimentação, driver para bobina de ignição (IGBT), alicate amperímetro, ponta de alta tensão, carga capacitiva/resistiva e uma bobina de ignição 1x1. Na Figura 4, pode-se encontrar o esquema simplificado do circuito de medição.

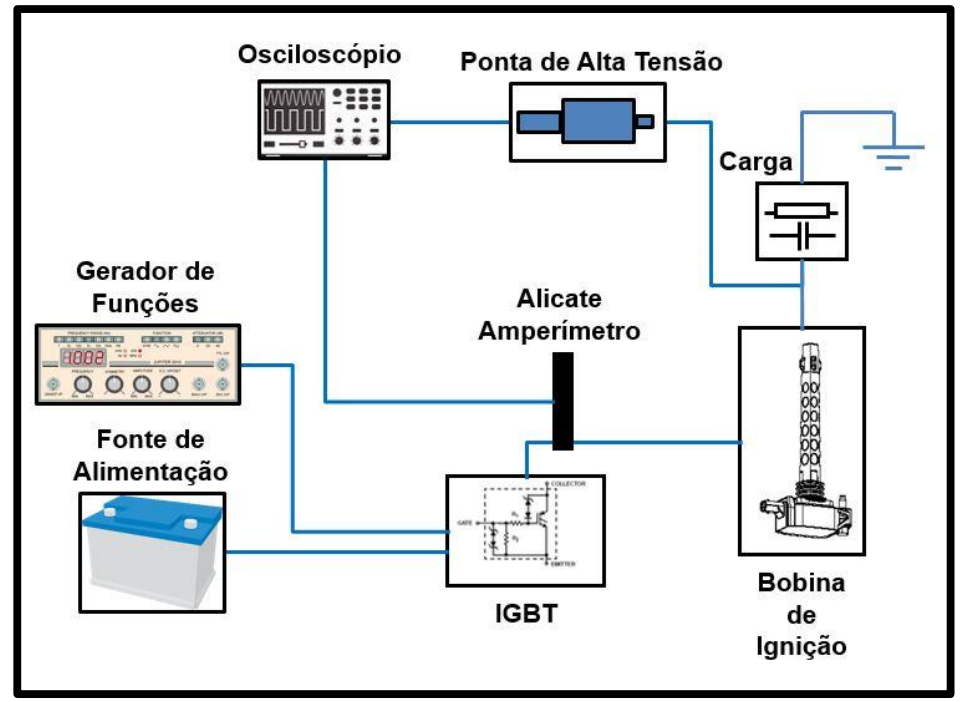

Figura 4. Esquema do circuito de medição para o ensaio de oferta de tensão. [Elaborado pelo autor] 
No sistema da Figura 4, o osciloscópio tem a função de adquirir as formas de onda provenientes das pontas de prova e executar as medições necessárias para avaliar o produto. A ponta de prova de alta tensão mede o nível de alta tensão ofertado na saída da bobina e o alicate amperímetro mede o nível de corrente aplicado no enrolamento primário.

O gerador de funções tem a finalidade de prover o sinal necessário para o acionamento do driver da bobina de ignição. Através do gerador é possível controlar a corrente elétrica do primário, variando-se o dwell time do pulso aplicado na entrada do IGBT (Gate).

A fonte de alimentação é responsável por fornecer a energia que o sistema demanda para pleno funcionamento, é primordial que a alimentação seja feita de forma adequada para que não ocorra interferência nas medições devido à limitação de energia pelo equipamento.

O driver da bobina de ignição é responsável por receber um pulso de entrada e então chavear o sinal de potência em seus terminais de saída, isso permite o carregamento do circuito primário da bobina e posterior conversão de energia.

Para exemplificar como é feita a conexão entre driver, bobina e carga, na Figura 5, podese observar em detalhes o circuito utilizado para acionamento das amostras para execução do ensaio de oferta de tensão.

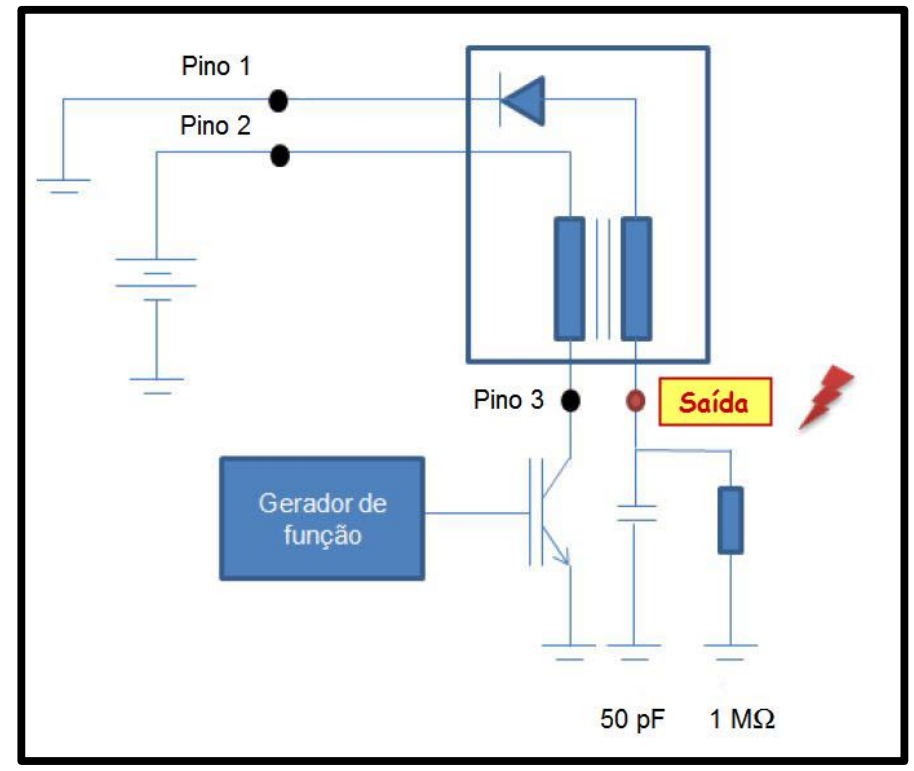

Figura 5. Circuito para ensaio de oferta de tensão. [6]

Com intuito de simular as condições encontradas em campo, a carga utilizada em bancada de testes possui dois componentes, um capacitivo e um resistivo. Dependendo da aplicação, os valores dos componentes, ou mesmo a presença de uma das partes, pode se alterar, dependendo da finalidade. O objetivo é simular em bancada, o mais fielmente possível, a carga encontrada em campo, que no caso da bobina de ignição, é a vela de ignição.

Os parâmetros de acionamento utilizados para execução do ensaio são apresentados na Tabela 1. 
Tabela 1. Parâmetros de acionamento para o ensaio de Oferta de Tensão. [6]

\begin{tabular}{|l|l|}
\hline Item & Descrição \\
\hline Estágio de potência & IGBT (driver externo) \\
\hline Tensão de bateria $(\mathrm{T})$ & $13,50 \mathrm{~V} \pm 0,05 \mathrm{~V}$ \\
\hline Resistência do Primário $\left(\mathrm{R}_{\mathrm{wh}}\right)$ & $100 \mathrm{~m} \Omega \pm 15 \mathrm{~m} \Omega$ \\
\hline Frequência de acionamento & $50 \mathrm{~Hz} \pm 1 \mathrm{~Hz}$ \\
\hline Tempo de carga $\left(\mathrm{t}_{\text {dwell }}\right)$ & $2,40 \mathrm{~ms} \pm 0,02 \mathrm{~ms}$ \\
\hline Carga do secundário $(\mathrm{F})$ & $50 \mathrm{pF} \pm 3 \mathrm{pF} / / 1 \mathrm{M} \Omega \pm 0,025 \mathrm{M} \Omega$ \\
\hline
\end{tabular}

Os critérios de aprovação utilizados para o ensaio são apresentados na Tabela 2.

Tabela 2. Critérios de aprovação para o ensaio de Oferta de Tensão. [6]

\begin{tabular}{|l|l|}
\hline Item & Descrição \\
\hline $\begin{array}{l}\text { Elevação da corrente do primário após } \\
\text { tempo de carga }\left(\mathrm{I}_{1}\right)\end{array}$ & $9,4 \mathrm{~A} \leq \mathrm{I}_{1}$ nom $\leq 10,6 \mathrm{~A}$ \\
\hline Tensão no secundário $\left(\mathrm{U}_{2}\right)$ & $28 \mathrm{kV} \leq\left|\mathrm{U}_{2} \max \right| \leq 33 \mathrm{kV}$ \\
\hline $\begin{array}{l}\text { Tempo de subida da tensão do } \\
\text { secundário }\left(\mathrm{t}_{\mathrm{r}}\right)\end{array}$ & $12 \mu \mathrm{s} \leq \mathrm{t}_{\mathrm{r}} \leq 16 \mu \mathrm{s}$ \\
\hline
\end{tabular}

A fim de esclarecer de maneira gráfica os principais parâmetros discutidos nesse artigo, nas Figuras 6 e 7, encontram-se as curvas características da corrente do primário e da oferta de tensão no secundário, respectivamente. Os parâmetros medidos são: o máximo valor da corrente do primário $\left(\mathrm{I}_{1}\right)$, o mínimo valor da oferta de tensão (pico negativo) $\left(\mathrm{U}_{2}\right)$ e o rise time (pico negativo) $\left(\mathrm{t}_{\mathrm{r}}\right)$.

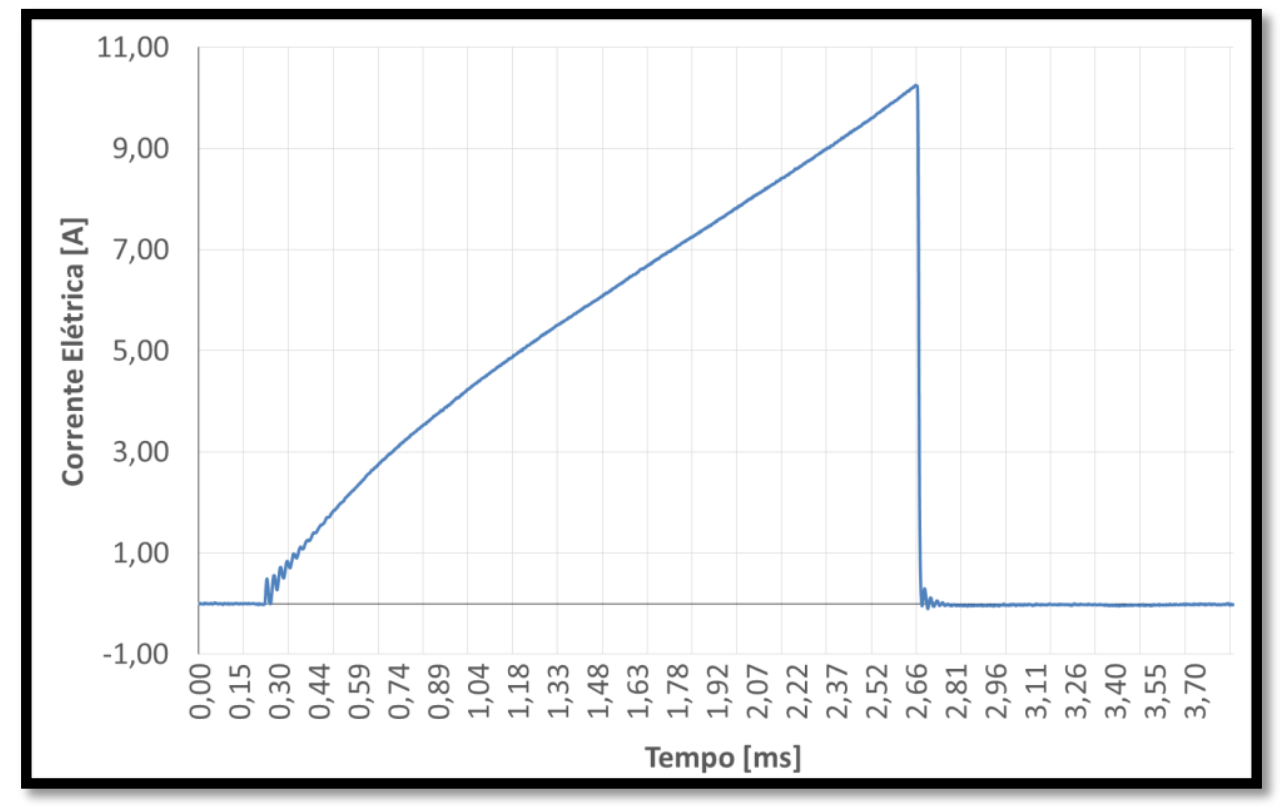

Figura 6. Curva Característica da Corrente do Primário $\left(\mathrm{I}_{1}\right)$. 
A elevação de corrente é controlada através do sinal do gerador de funções, aplicado ao IGBT, e o valor máximo de corrente varia de acordo com o tempo em $O N$ do sinal, o que permite o controle preciso da energia carregada no enrolamento primário. Ao atingir o nível de energia desejado, a corrente é levada a zero e a energia é transferida para a saída do componente.

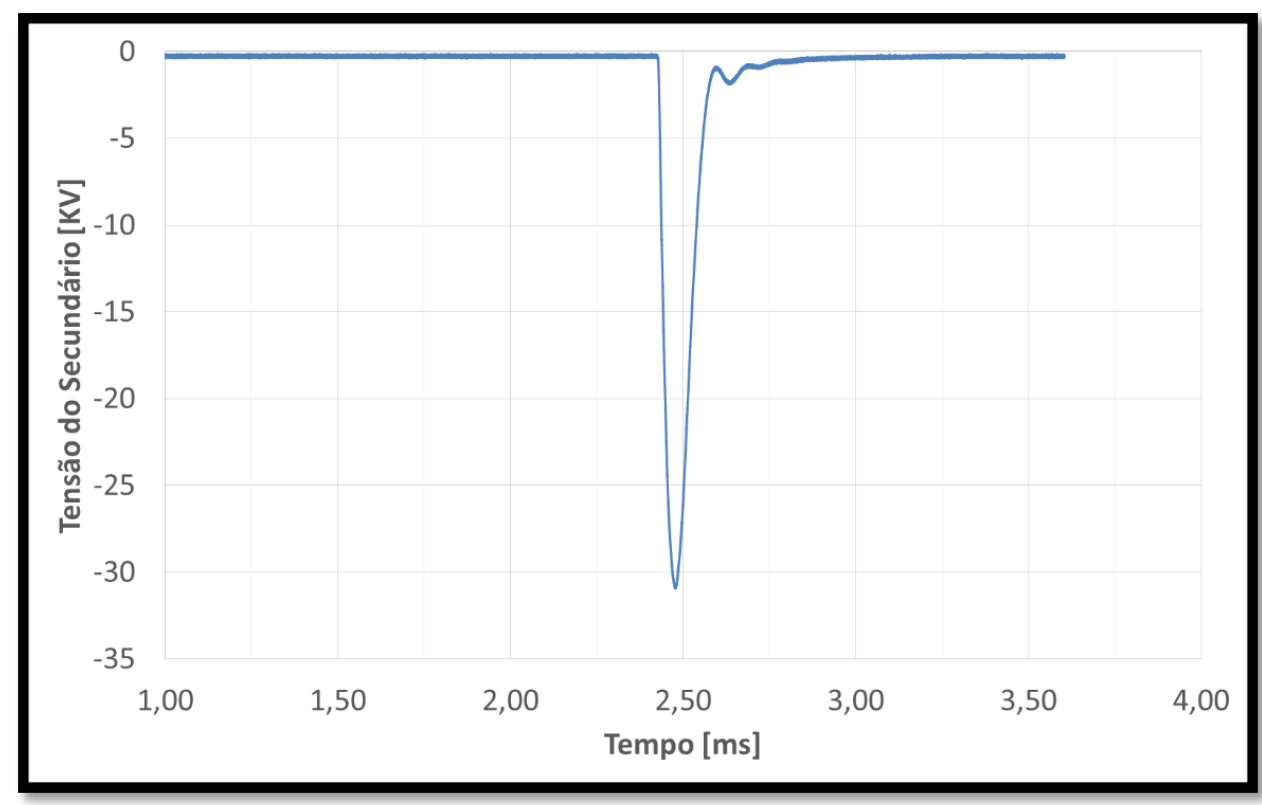

Figura 7. Curva Característica da Tensão do Secundário $\left(\mathrm{U}_{2}\right)$.

A tensão do secundário é efeito da relação de transformação do componente. Utilizando uma carga capacitiva em paralelo com uma carga resistiva a curva característica tem o formato mostrado na Figura 7. A curva observada em bancada de testes difere da curva medida em veículo, pois utilizando a vela de ignição existe a centelha, que tem o comportamento diferente ao da curva com carga simulada em bancada.

\subsection{Ensaio de Energia da Centelha}

Assim como no ensaio de oferta de tensão, o objetivo do ensaio de energia é verificar a performance da bobina de ignição, analisando a energia transferida para o enrolamento secundário $\left(\mathrm{W}_{\mathrm{sp}}\right)$, a corrente de saída $\left(\mathrm{I}_{\mathrm{sp}}\right)$ e a duração da faísca $\left(\mathrm{t}_{\mathrm{sp}}\right)$. O procedimento consiste em aplicar um sinal de potência nos terminais de entrada do componente, medir a energia da faísca, a duração da faísca e a corrente de pico na saída do circuito secundário. Esses parâmetros são avaliados conforme requisitos previamente estabelecidos e, então, uma decisão pode ser tomada levando-os em consideração.

O ensaio foi realizado na bancada de performance de bobina de ignição no laboratório da engenharia de testes. Os equipamentos de teste utilizados para a execução das medições foram: osciloscópio, gerador de funções, fonte de alimentação, driver para bobina de ignição (IGBT), alicate amperímetro, amplificador de sinais, sistema de medição de corrente para pequenos sinais, ponta de alta tensão, banco de diodos Zener e uma bobina de ignição 1x1. Na Figura 8, pode-se encontrar o esquema simplificado do circuito de medição. 


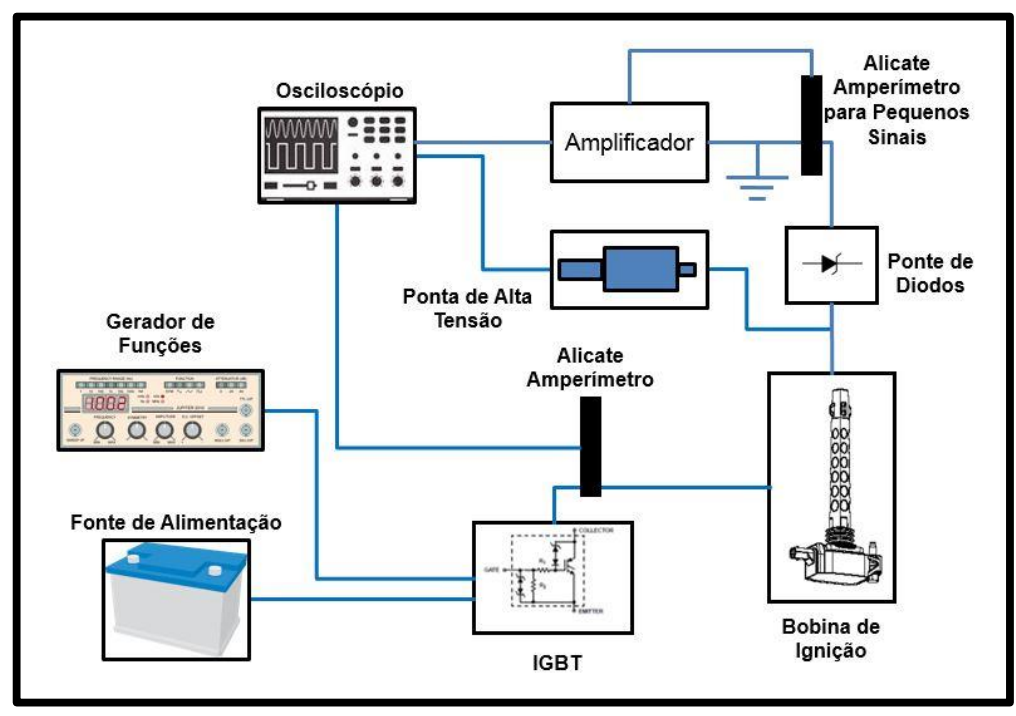

Figura 8. Circuito para o ensaio de Energia. [Elaborado pelo autor]

No sistema da Figura 8, a ponta de prova de alta tensão mede a tensão de grampeamento proveniente da ponte de diodos, o sistema de medição de corrente para pequenos sinais é responsável por medir a corrente do circuito secundário e o alicate amperímetro mede a corrente do circuito primário.

Para medições desse tipo, utiliza-se um amplificador juntamente com uma ponta de prova, para que seja possível executar a medição com a resolução adequada, ou seja, como a corrente da faísca é muito pequena, é necessário um sistema de medição avançado para adquirir adequadamente o sinal desejado, assim, é possível medições com níveis de até $1 \mathrm{~mA}$.

Para exemplificar como é feita a conexão entre o driver, a bobina e a carga, na Figura 9 encontra-se, em detalhes, o circuito utilizado para acionamento das amostras para execução do ensaio de energia da faísca.

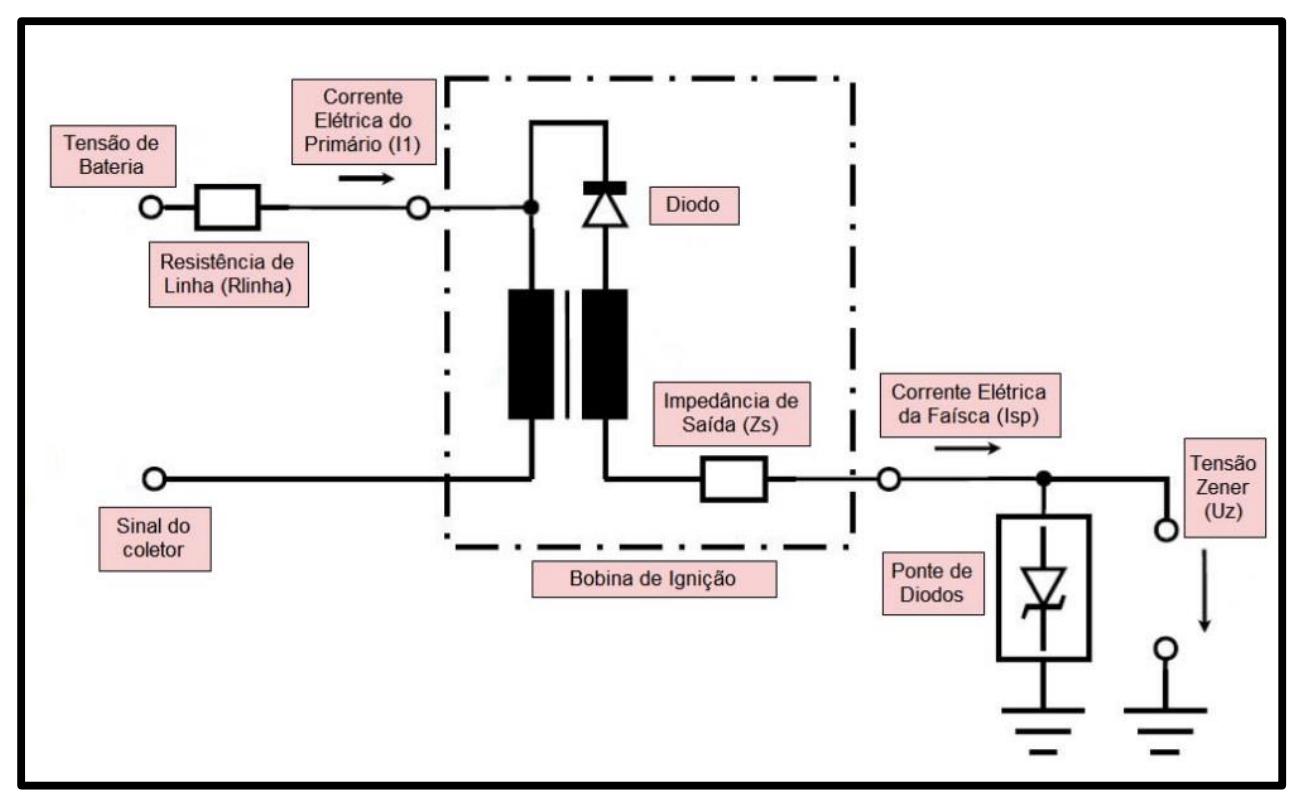

Figura 9. Circuito para Medição de Energia da Faísca. 
A carga utilizada para o ensaio de energia da faísca é um banco de diodos. Através dele, é possível grampear a tensão de saída da bobina em diferentes níveis de tensão. Para o ensaio aqui discutido, o grampeamento foi feito em $1000 \mathrm{~V}$, ou seja, a bobina tem como carga um banco de diodos que irá fixar a tensão de saída em $1000 \mathrm{~V}$. Dessa maneira, é possível avaliar como a bobina se comportará em campo, pois a maior parte da energia desprendida da bobina é verificada no tempo de queima, que ocorre posteriormente ao evento da ruptura do dielétrico, então, é possível avaliar se a bobina terá energia suficiente para que ocorra a queima adequada da mistura ar e combustível.

A medição da energia da faísca é realizada utilizando como carga um conjunto de diodos simulando a tensão de queima da faísca. Desta forma a tensão Zener é a soma das tensões de cada diodo e da parcela de um resistor de $100 \Omega$ em série com o conjunto de diodos. A energia será verificada através da integral do produto da tensão Zener e da corrente do secundário relativa ao tempo, cálculo realizado através do osciloscópio.

Os parâmetros de acionamento utilizados para execução do ensaio de Energia são apresentados na Tabela 3.

Tabela 3. Parâmetros de acionamento para o ensaio de Energia. [6]

\begin{tabular}{|l|l|}
\hline Item & Descrição \\
\hline Estágio de potência & IGBT (driver externo) \\
\hline Tensão de bateria $(\mathrm{T})$ & $13,50 \mathrm{~V} \pm 0,05 \mathrm{~V}$ \\
\hline Resistência do Primário $\mathrm{R}_{\mathrm{wh}}$ & $100 \mathrm{~m} \Omega \pm 15 \mathrm{~m} \Omega$ \\
\hline Frequência de acionamento & $50 \mathrm{~Hz} \pm 1 \mathrm{~Hz}$ \\
\hline Tempo de carga $\left(\mathrm{t}_{\text {dwell }}\right)$ & $2,40 \mathrm{~ms} \pm 0,02 \mathrm{~ms}$ \\
\hline Carga do secundário $\left(\mathrm{U}_{\mathrm{z}}\right)$ & $1000 \mathrm{Vz} \pm 50 \mathrm{Vz}$ \\
\hline
\end{tabular}

Os critérios de aprovação utilizados para o ensaio de Energia são apresentados na Tabela 4.

Tabela 4. Critérios de aprovação para o ensaio de oferta de tensão. [6]

\begin{tabular}{|l|l|}
\hline Item & Descrição \\
\hline Energia da centelha $\left(\mathrm{W}_{\mathrm{sp}}\right)$ & $>58 \mathrm{~mJ}$ \\
\hline Corrente da centelha $\left(\left|\mathrm{I}_{\mathrm{sp}}\right|\right)$ & $<100 \mathrm{~mA}$ \\
\hline Duração da centelha $\left(\mathrm{t}_{\mathrm{sp}}\right)$ & $\geq 1,45 \mathrm{~ms}$ \\
\hline
\end{tabular}

Nas Figuras 10 e 11, encontram-se as curvas características da corrente do secundário e da tensão grampeada pelo banco de diodos (Tensão Zener), respectivamente. Os parâmetros medidos são: o máximo valor da corrente do secundário ( $\left.\mathrm{I}_{\mathrm{SP}}\right)$, a energia da centelha ( $\left.\mathrm{W}_{\mathrm{SP}}\right)$ e a duração da centelha ( $\left.\mathrm{t}_{\mathrm{SP}}\right)$. 


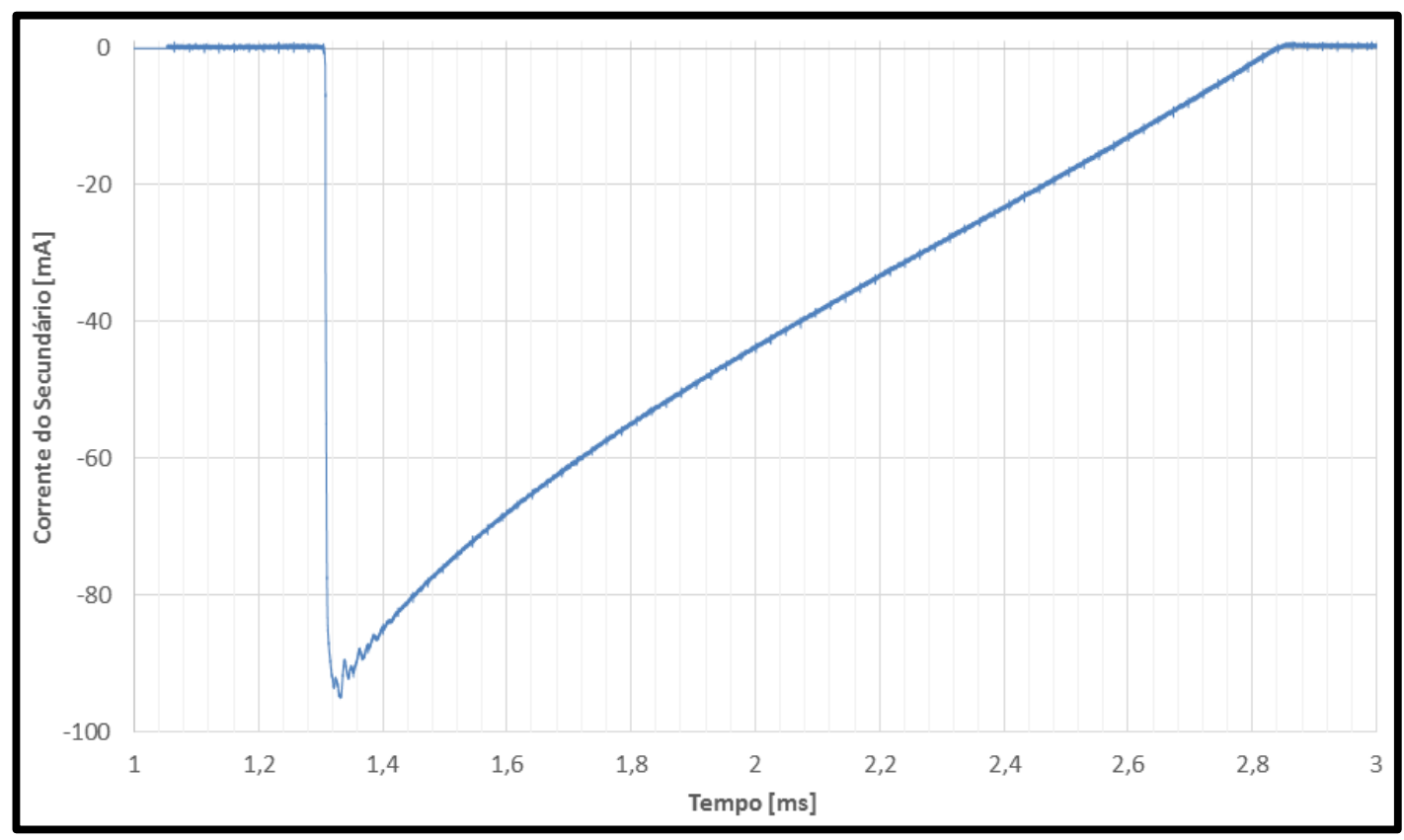

Figura 10. Curva Característica da Corrente do Secundário ( $\left.\mathrm{I}_{\mathrm{SP}}\right)$.

A corrente do secundário é reflexo da força eletromotriz (FEM) induzida no enrolamento secundário. A FEM, é gerada pela variação de fluxo magnético no enrolamento primário. Assim como a corrente do primário, a corrente do secundário pode ser controlada através do sinal do gerador de funções, aplicado ao IGBT, uma vez que ambas correntes são diretamente proporcionais. $\mathrm{O}$ valor máximo da corrente do secundário varia de acordo com o tempo em $O N$ do sinal, o que permite o controle preciso da energia de saída do sistema. A corrente pode ser verificada até o fim do tempo de queima. Assim sendo, ela é gradativamente decrementada, até que toda a energia tenha sido desprendida durante a queima da mistura do cilindro.

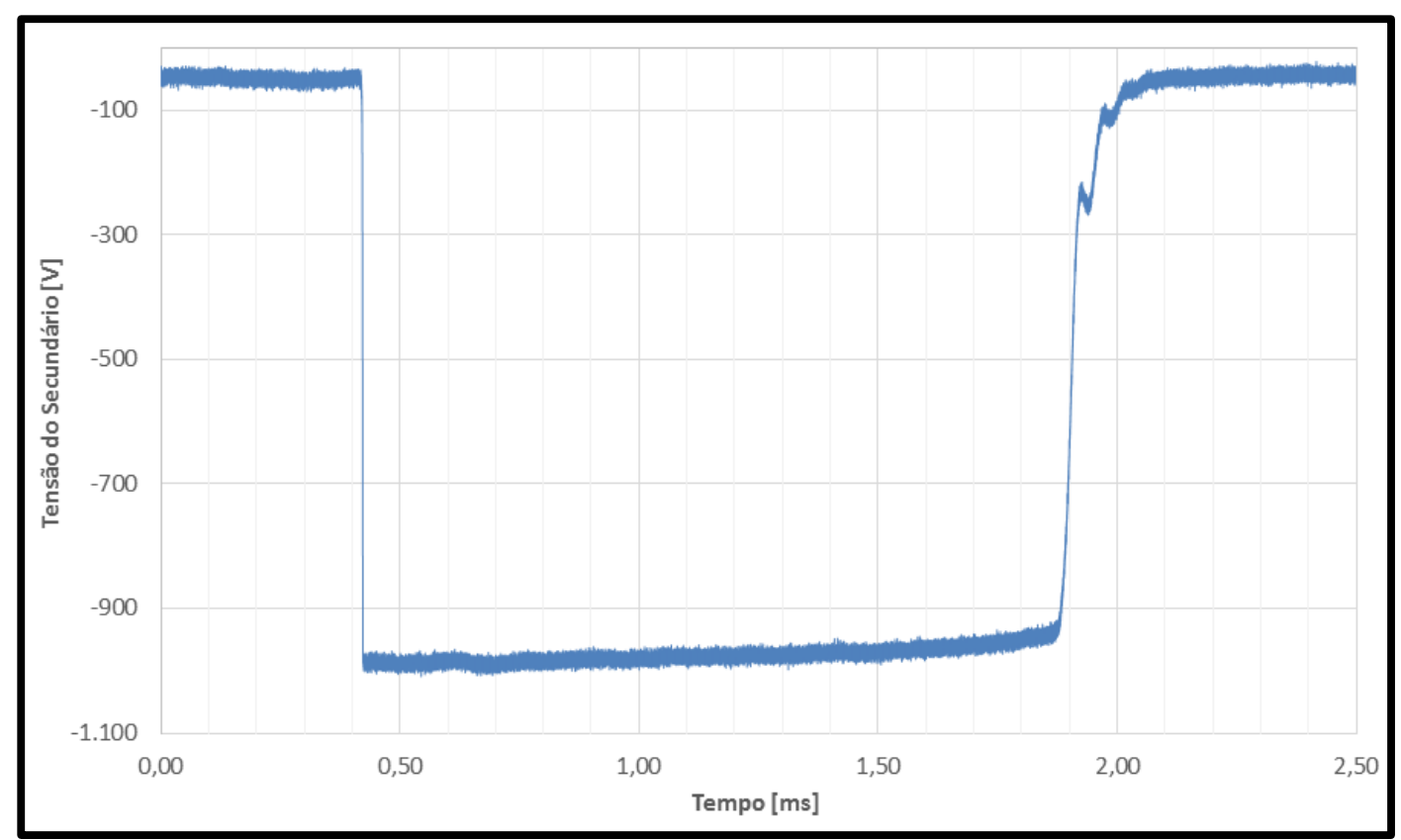

Figura 11. Curva Característica da tensão Zener. 
A tensão grampeada no enrolamento secundário tem o formato apresentado na Figura 11. Ela possui essa forma característica pois, a ponte de diodos utilizada como carga do sistema, tem a função de fixar o valor da tensão do secundário. Isso é feito a fim de simular o evento após a ruptura do dielétrico, denominado tempo de queima. A carga utilizada para os ensaios de energia varia de acordo com a aplicação, porém sempre se utiliza a ponte de diodos para grampear o valor de tensão de saída. Algumas aplicações demandam uma componente capacitiva e uma componente resistiva em paralelo com a ponte de diodos.

\subsection{Relações de Causa e Efeito}

Para o trabalho proposto, utilizou-se uma amostra referência, no qual é utilizada como padrão de estabilidade das medições.

Todas os procedimentos foram realizados em ambiente com temperatura controlada, $\mathrm{o}$ monitoramento da temperatura ambiente é pré-requisito para esse teste, portanto, foi mantida em $23^{\circ} \mathrm{C} \pm 3^{\circ} \mathrm{C}$. Para esse ensaio, foram executadas medições na mesma amostra, ou seja, os parâmetros de entrada do sistema foram alterados um por vez, para que as relações de causa e efeito pudessem ser identificadas adequadamente. Assim, para cada variação de um parâmetro de entrada, foi verificada a variação em cada grandeza de saída e foi estabelecida uma relação entre elas.

Logo, variou-se os parâmetros de tensão de entrada, capacitância, tensão Zener e $d w e l l$ time, após, foram medidas as variações na saída, obtendo-se a relação entre as grandezas de entrada e de saída. A partir desta relação, obtivera-se, por meio de regressões lineares ou polinomiais e com auxílio do software Microsoft Excel@, as equações matemáticas no qual descrevem tal relação.

Desta forma, foram obtidas 18 equações de relação: tensão de entrada $x U_{2}$, tensão de entrada $\times \mathrm{I}_{1}$, tensão de entrada $\times \mathrm{t}_{\mathrm{r}}$, tensão de entrada $\times \mathrm{W}_{\mathrm{SP}}$, tensão de entrada $\times \mathrm{I}_{\mathrm{SP}}$, tensão de entrada $\mathrm{x} \mathrm{t}_{\mathrm{SP}}$, dwell time $\mathrm{x} \mathrm{U}_{2}$, dwell time $\mathrm{x}_{1}$, dwell time $\mathrm{x}_{\mathrm{r}}$, dwell time $\mathrm{x} \mathrm{W}_{\mathrm{SP}}$, dwell time $\mathrm{x} \mathrm{I}_{\mathrm{SP}}$, dwell time $\mathrm{x} \mathrm{t}_{\mathrm{SP}}$, capacitância $\mathrm{x} \mathrm{U}_{2}$, capacitância $\mathrm{x} \mathrm{I}_{1}$, capacitância $\times \mathrm{t}_{\mathrm{r}}$, tensão Zener x $\mathrm{W}_{\mathrm{SP}}$, tensão Zener $\mathrm{x} \mathrm{I}_{\mathrm{SP}}$ e tensão Zener $\mathrm{x}$ tsP.

A tensão de entrada, o tempo de carga (dwell time), a carga do secundário (capacitância) e a tensão Zener, foram variadas em torno do valor nominal da aplicação, com intervalos que permitiram observar variações nas grandezas de saída.

\section{RESULTADOS E DISCUSSÃO}

A seguir são apresentados os resultados obtidos através dos testes executados na bancada de ensaios funcionais de bobina de ignição, no centro de testes de engenharia da Robert Bosch Ltda.

\subsection{Ensaio de Oferta de Tensão}

Como mencionado no item "materiais e métodos", foram variados as grandezas de entrada e observados as variações nas grandezas de saída. Para o ensaio de oferta de tensão, variaram-se a tensão de entrada $(\mathrm{T})$, a carga $(\mathrm{F})$ e o dwell time $\left(\mathrm{t}_{\mathrm{dwell}}\right)$, e observouse a variação na tensão do secundário $\left(\mathrm{U}_{2}\right)$, corrente do primário $\left(\mathrm{I}_{1}\right)$ e rise time $\left(\mathrm{t}_{\mathrm{r}}\right)$. 
Abaixo, na Figura 12, pode-se observar o gráfico da relação entre a tensão de entrada $(T)$ e a Tensão do secundário $\left(\mathrm{U}_{2}\right)$.

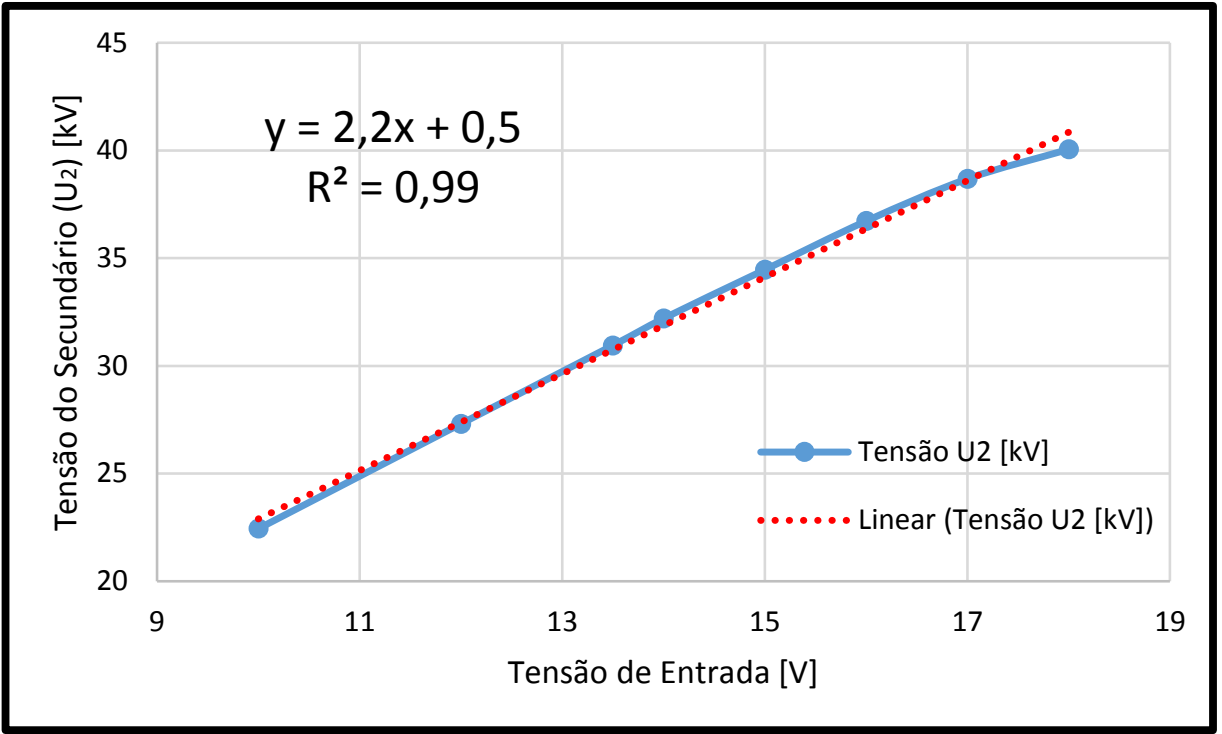

Figura 12. Gráfico $\mathrm{T}$ x $\mathrm{U}_{2}$

Na Tabela 5, pode-se observar os valores obtidos nas grandezas de saída quando variado a tensão de entrada.

Tabela 5: Variação da tensão de entrada no ensaio de oferta de tensão ( $\mathrm{t}_{\mathrm{dwell}}=2,4 \mathrm{~ms} ; \mathrm{F}=50 \mathrm{pF} / / 1 \mathrm{M} \Omega$ ).

\begin{tabular}{c|ccc} 
Tensão de Entrada $[\mathbf{V}]$ & Corrente $\left(\mathbf{I}_{\mathbf{1}}\right)[\mathbf{A}]$ & Tensão $\left(\mathbf{U}_{\mathbf{2}}\right)[\mathbf{k V}]$ & Rise time $\left(\mathbf{t}_{\mathbf{r}}\right)[\boldsymbol{\mu} \mathbf{s}]$ \\
\hline $\mathbf{1 0 , 0 0}$ & 7,05 & 22,44 & 26,15 \\
$\mathbf{1 2 , 0 0}$ & 8,73 & 27,31 & 18,12 \\
$\mathbf{1 3 , 5 0}$ & 10,28 & 30,95 & 14,85 \\
$\mathbf{1 4 , 0 0}$ & 11,01 & 32,20 & 13,82 \\
$\mathbf{1 5 , 0 0}$ & 12,87 & 34,46 & 11,97 \\
$\mathbf{1 6 , 0 0}$ & 15,34 & 36,72 & 10,40 \\
$\mathbf{1 7 , 0 0}$ & 18,28 & 38,68 & 9,13 \\
$\mathbf{1 8 , 0 0}$ & 21,47 & 40,05 & 8,37
\end{tabular}

Observa-se que, de acordo com o incremento da tensão de entrada a tensão de saída e a corrente tendem a aumentar, o que já era esperado, pois as relações são diretamente proporcionais, ou seja, quanto maior a tensão de entrada, mais energia se acumula no enrolamento primário, o que significa aumento no pico de corrente e consequentemente aumento da FEM induzida no enrolamento secundário, as relações podem ser observadas na equação 3 e na equação 4 .

Por outro lado, o rise time tende a diminuir, uma vez que esse parâmetro é medido em pontos fixos da curva da tensão do secundário, então aumentando a tensão de entrada, a centelha torna-se mais veloz, e consequentemente o tempo de subida da faísca diminui.

Na Tabela 6, observa-se os valores obtidos no ensaio de oferta de tensão quando variado a carga do secundário. 
Tabela 6: Variação da carga do secundário no ensaio de oferta de tensão $\left(\mathrm{T}=13,5 \mathrm{~V} ; \mathrm{t}_{\mathrm{dwell}}=2,4 \mathrm{~ms}\right)$.

\begin{tabular}{c|ccc} 
Capacitância $[\mathbf{p F}]$ & Corrente $\left(\mathbf{I}_{\mathbf{1}}\right)[\mathbf{A}]$ & Tensão $\left(\mathbf{U}_{\mathbf{2}}\right)[\mathbf{k V}]$ & Rise time $\left(\mathbf{t}_{\mathbf{r}}\right)[\boldsymbol{\mu s}]$ \\
\hline $\mathbf{1 7 , 0 0}$ & 10,26 & 36,75 & 7,99 \\
$\mathbf{2 0 , 0 0}$ & 10,27 & 35,90 & 8,59 \\
$\mathbf{3 0 , 0 0}$ & 10,27 & 33,86 & 10,59 \\
$\mathbf{4 0 , 0 0}$ & 10,29 & 32,22 & 12,70 \\
$\mathbf{5 0 , 0 0}$ & 10,24 & 30,75 & 14,96 \\
$\mathbf{6 0 , 0 0}$ & 10,29 & 29,64 & 17,05 \\
$\mathbf{7 0 , 0 0}$ & 10,31 & 28,49 & 19,11 \\
$\mathbf{8 0 , 0 0}$ & 10,30 & 27,49 & 21,27
\end{tabular}

Em relação a variação da capacitância, pode-se notar que a corrente permanece constante, pois ela não depende da carga que a bobina de ignição está submetida, e sim apenas de parâmetros que estão relacionados ao enrolamento primário, ver equação 3.

Já a tensão, tende a diminuir de acordo com o aumento da capacitância, pois diminui-se a queda de tensão sobre a carga.

Em contrapartida, o rise time aumenta com o incremento da capacitância. Esse parâmetro é medido em pontos fixos da curva da tensão do secundário, então aumentando-se a capacitância, a centelha torna-se mais lenta, e consequentemente o tempo de subida da faísca aumenta.

Na Tabela 7, observa-se os valores obtidos no ensaio de oferta de tensão quando variado o tempo de carregamento do primário.

Tabela 7: Variação do dwell time no ensaio de oferta de tensão (T = 13,5 V; F = 50pF // 1M $\Omega$ ).

\begin{tabular}{c|ccc} 
Dwell Time $[\mathrm{ms}]$ & Corrente $\left(\mathbf{I}_{\mathbf{1}}\right)[\mathbf{A}]$ & Tensão $\left(\mathbf{U}_{\mathbf{2}}\right)[\mathbf{k V}]$ & Rise time $\left(\mathbf{t}_{\mathbf{r}}\right)[\boldsymbol{\mu s}]$ \\
\hline $\mathbf{1 , 6 0}$ & 7,39 & 23,67 & 22,85 \\
$\mathbf{2 , 0 0}$ & 8,78 & 27,61 & 17,80 \\
$\mathbf{2 , 4 0}$ & 10,33 & 31,15 & 14,69 \\
$\mathbf{2 , 8 0}$ & 12,34 & 34,12 & 12,34 \\
$\mathbf{3 , 2 0}$ & 14,48 & 36,19 & 10,79 \\
$\mathbf{3 , 6 0}$ & 16,02 & 37,40 & 9,97 \\
$\mathbf{4 , 0 0}$ & 16,89 & 37,95 & 9,61
\end{tabular}

De acordo com a Tabela 7, nota-se que com o incremento do dwell time, a corrente do primário e a tensão de saída tendem a aumentar, pois quanto maior o tempo de carga, maior será a energia acumulada no enrolamento primário, isso significa uma corrente de pico maior. Consequentemente, a variação de fluxo magnético será maior, assim a FEM induzida no lado secundário também aumentará, ver equação 1.

Já, o rise time, tende a diminuir, uma vez que esse parâmetro é medido em pontos fixos da curva da tensão do secundário, então aumentando o dwell time, a centelha torna-se mais veloz, e consequentemente o tempo de subida da faísca diminui.

A partir desta base de dados, foram plotados gráficos correlacionando cada uma das grandezas de entrada pelas grandezas de saída. Após, por meio da regressão do software Microsoft Excel@, obtiveram-se as equações nos quais descrevem tais relações. Cabe 
ressaltar que, tais equações são válidas apenas para a faixa de entrada das grandezas abordadas neste estudo, para outros valores o comportamento da bobina pode ser diferente ao observado neste trabalho.

A partir dos gráficos, obtivera-se as equações de relação entre as grandezas de entrada e saída, para o ensaio de oferta de tensão. As equações obtidas podem ser observadas na Tabela 8.

Tabela 8. Equações obtidas para as relações entre as grandezas de entrada e de saída para o ensaio de oferta de tensão e seus respectivos coeficiêntes de discriminação.

\begin{tabular}{c|cc}
\multicolumn{1}{c}{ Parâmetro } & Equação da Curva & Coeficiente de Discriminação \\
\hline \multirow{2}{*}{ Corrente $\left(\mathbf{I}_{\mathbf{1}}\right)[\mathbf{A}]$} & $\mathrm{I}_{1}=10,28$ & - \\
& $\mathrm{I}_{1}=4,2 \cdot \mathrm{t}_{\text {dwell }}+0,5$ & $\mathrm{R}^{2}=0,99$ \\
& $\mathrm{I}_{1}=0,2 \cdot \mathrm{T}^{2}-3,9 \cdot \mathrm{T}+25,7$ & $\mathrm{R}^{2}=0,99$ \\
Tensão de Saída & $\mathrm{U}_{2}=0,001 \cdot \mathrm{F}^{2}-0,252 \cdot \mathrm{F}+40,6$ & $\mathrm{R}^{2}=0,99$ \\
$\left(\mathbf{U}_{\mathbf{2}}\right)[\mathbf{k} \mathbf{V}]$ & $\mathrm{U}_{2}=-2,3 \cdot \mathrm{t}_{\mathrm{dwell}}{ }^{2}+18,7 \cdot \mathrm{t}_{\text {dwell }}-0,6$ & $\mathrm{R}^{2}=0,99$ \\
& $\mathrm{U}_{2}=2,2 \cdot \mathrm{T}+0,5$ & $\mathrm{R}^{2}=0,99$ \\
Rise Time $\left(\mathbf{t}_{\mathbf{r}}\right)[\boldsymbol{\mu} \mathbf{s}]$ & $\mathrm{t}_{\mathrm{r}}=0,2 \cdot \mathrm{F}+4,3$ & $\mathrm{R}^{2}=0,99$ \\
& $\mathrm{t}_{\mathrm{r}}=2,7 \cdot \mathrm{t}_{\mathrm{dwell}}{ }^{2}-20,5 \cdot \mathrm{t}_{\mathrm{dwell}}+48,4$ & $\mathrm{R}^{2}=0,99$ \\
& $\mathrm{t}_{\mathrm{r}}=0,2 \cdot \mathrm{T}^{2}-8,3 \cdot \mathrm{T}+87,1$ & $\mathrm{R}^{2}=0,99$
\end{tabular}

OBS.: Capacitância (F) em pF, Tensão de entrada $(\mathrm{T})$ em $\mathrm{V}$ e dwell time $\left(\mathrm{t}_{\mathrm{dwell}}\right)$ em ms.

Nota-se que, todas as equações obtidas por meio do software Microsoft Excel ${ }^{\circledR}$ obtiveram um bom coeficiente de discriminação $\left(R^{2} \geq 0,99\right)$, ou seja, as equações representam o comportamento observado em bancada.

\subsection{Ensaio de Energia}

Para o ensaio de energia, variaram-se a tensão de entrada $(T)$, a tensão Zener $\left(U_{z}\right)$ e o dwell time $\left(\mathrm{t}_{\mathrm{dwell}}\right)$, e observou-se a variação na Energia da centelha $\left(\mathrm{W}_{\mathrm{SP}}\right)$, corrente do secundário ( $\left.\mathrm{I}_{\mathrm{SP}}\right)$ e tempo da centelha $\left(\mathrm{t}_{\mathrm{SP}}\right)$.

Abaixo, na Figura 11, pode-se observar o gráfico da relação entre a tensão de entrada e a corrente do secundário.

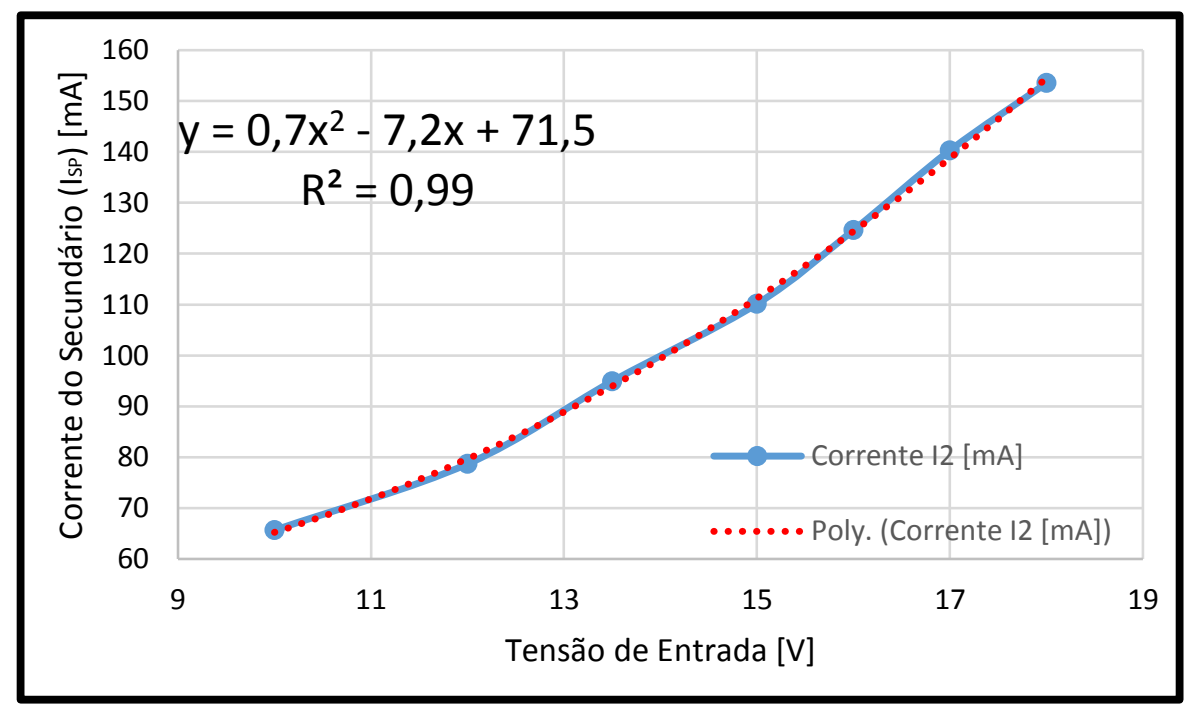

Figura 13. Gráfico T x I $\mathrm{SP}$ 
Na Tabela 9, pode-se observar os valores obtidos nas grandezas de saída, quando variado a tensão de entrada.

Tabela 9: Variação da tensão de entrada no ensaio de Energia. $\left(t_{\text {dwell }}=2,4 \mathrm{~ms} ; \mathrm{U}_{\mathrm{z}}=1000 \mathrm{~V}\right)$.

\begin{tabular}{c|ccc} 
Tensão de Entrada [V] & $\begin{array}{c}\text { Corrente }\left(\mathbf{I s p}_{\mathbf{S P}}\right) \\
{[\mathbf{m A}]}\end{array}$ & $\begin{array}{c}\text { Energia }\left(\mathbf{W}_{\mathbf{s p}}\right) \\
{[\mathbf{m J}]}\end{array}$ & $\begin{array}{c}\text { Duração da Centelha }\left(\mathbf{t}_{\mathbf{s p}}\right) \\
{[\mathbf{m s}]}\end{array}$ \\
\hline $\mathbf{1 0 , 0 0}$ & 65,64 & 36,39 & 1,20 \\
$\mathbf{1 2 , 0 0}$ & 78,67 & 47,95 & 1,32 \\
$\mathbf{1 3 , 5 0}$ & 94,92 & 65,21 & 1,42 \\
$\mathbf{1 5 , 0 0}$ & 110,13 & 76,91 & 1,67 \\
$\mathbf{1 6 , 0 0}$ & 124,63 & 83,72 & 1,73 \\
$\mathbf{1 7 , 0 0}$ & 140,30 & 89,94 & 1,77 \\
$\mathbf{1 8 , 0 0}$ & 153,50 & 94,03 & 1,80
\end{tabular}

Observa-se que, de acordo com o incremento da tensão de entrada, todos os parâmetros medidos tendem a aumentar, o que já era esperado, devido ao acúmulo de energia no primário ser maior, assim transfere-se mais energia ao secundário. Dessa maneira, a corrente do secundário, a energia da centelha e a duração da centelha também aumentam.

Na Tabela 10, observa-se os valores obtidos no ensaio de Energia, quando variado a carga do secundário.

Tabela 10: Variação da carga do secundário no ensaio de Energia $\left(\mathrm{t}_{\mathrm{dwell}}=2,4 \mathrm{~ms} ; \mathrm{T}=13,5 \mathrm{~V}\right)$.

\begin{tabular}{c|ccc}
$\begin{array}{c}\text { Tensão Zener }\left(\mathbf{U}_{\mathbf{z}}\right) \\
{[\mathbf{V}]}\end{array}$ & $\begin{array}{c}\text { Corrente }(\mathbf{I} \mathbf{s P}) \\
{[\mathbf{m A}]}\end{array}$ & $\begin{array}{c}\text { Energia }\left(\mathbf{W}_{\mathbf{s p}}\right) \\
{[\mathbf{m J}]}\end{array}$ & $\begin{array}{c}\text { Duração da Centelha }\left(\mathbf{t}_{\mathbf{s p}}\right) \\
{[\mathbf{m s}]}\end{array}$ \\
\hline $\mathbf{4 5 0 , 0 0}$ & 96,80 & 48,91 & 2,77 \\
$\mathbf{6 5 0 , 0 0}$ & 96,04 & 55,46 & 2,27 \\
$\mathbf{8 0 0 , 0 0}$ & 95,59 & 61,86 & 1,86 \\
$\mathbf{1 0 0 0 , 0 0}$ & 95,15 & 65,39 & 1,56 \\
$\mathbf{2 0 0 0 , 0 0}$ & 93,29 & 75,23 & 0,93
\end{tabular}

Em relação a variação da tensão de grampeamento, nota-se que o valor da corrente e o valor da duração da centelha diminuem, pois, ao aumentar a tensão de grampeamento a impedância da carga tem um leve incremento, o que diminui sutilmente a corrente do secundário. Já a duração da faísca diminui, pois, a tensão de saída é maior, então o tempo de queima também diminui.

Por outro lado, a energia da centelha tende a aumentar pois o valor da tensão de saída é maior.

Na Tabela 11, observa-se os valores obtidos no ensaio de Energia quando variado o tempo de carregamento. 
Tabela 11: Variação do dwell time de entrada no ensaio de Energia $\left(\mathrm{U}_{\mathrm{z}}=1000 \mathrm{~V} ; \mathrm{T}=13,5 \mathrm{~V}\right)$.

\begin{tabular}{|c|c|c|c|}
\hline Dwell Time $[\mathrm{ms}]$ & $\begin{array}{c}\text { Corrente }\left(\mathbf{I}_{2}\right) \\
{[\mathbf{m A}]}\end{array}$ & $\begin{array}{c}\text { Energia }\left(W_{\text {sp }}\right) \\
{[\mathrm{mJ}]}\end{array}$ & Duração da Faísca $\left(t_{\mathrm{sp}}\right)[\mathrm{ms}]$ \\
\hline 1,60 & 70,34 & 41,09 & 1,25 \\
\hline 2,00 & 82,41 & 53,20 & 1,41 \\
\hline 2,40 & 95,25 & 65,69 & 1,55 \\
\hline 2,80 & 107,50 & 76,07 & 1,64 \\
\hline 3,20 & 119,97 & 83,32 & 1,70 \\
\hline 3,60 & 127,67 & 88,35 & 1,73 \\
\hline 4,00 & 133,90 & 90,38 & 1,75 \\
\hline
\end{tabular}

De acordo com a Tabela 11, nota-se que com o incremento do dwell time, todas as características medidas tendem a aumentar, devido ao acúmulo de energia do primário ser maior, assim transfere-se mais energia ao secundário. Dessa maneira, a corrente do secundário, a energia da centelha e a duração da centelha também aumentam

A partir destes dados, foram plotados os gráficos da relação entre as grandezas de entrada e saída. Após, assim como no ensaio de Oferta de Tensão, obtiveram-se as equações nos quais descrevem tais relações, por meio da regressão do software Microsoft Excel®.

A partir dos gráficos, obtivera-se as equações de relação entre as grandezas de entrada e saída para o ensaio de oferta de tensão. As equações obtidas podem ser observadas na Tabela 12.

Tabela 12. Equações obtidas para as relações entre as grandezas de entrada e de saída para o ensaio de Energia e seus respectivos coeficientes de discriminação.

\begin{tabular}{|c|c|c|}
\hline Parâmetro & Equação da Curva & $\begin{array}{l}\text { Coeficiente de } \\
\text { Discriminação }\end{array}$ \\
\hline \multirow{3}{*}{$\begin{array}{c}\text { Corrente (IsP) } \\
{[\mathbf{m A}]}\end{array}$} & $\mathrm{I}_{\mathrm{SP}}=8 \cdot 10^{-7} \cdot \mathrm{U}_{\mathrm{z}}^{2}-0,004 \cdot \mathrm{U}_{\mathrm{z}}+98,5$ & $\mathrm{R}^{2}=0,99$ \\
\hline & $\mathrm{ISP}_{\mathrm{SP}}=-4,1 \cdot t_{\text {dwell }}{ }^{2}+50 \cdot t_{\text {dwell }}-0,4$ & $\mathrm{R}^{2}=0,99$ \\
\hline & $\mathrm{I}_{\mathrm{SP}}=0,7 \cdot \mathrm{T}^{2}-7,2 \cdot \mathrm{T}+71,5$ & $\mathrm{R}^{2}=0,98$ \\
\hline \multirow{3}{*}{$\begin{array}{c}\text { Energia }\left(W_{S P}\right) \\
{[\mathbf{m J}]}\end{array}$} & $\mathrm{W}_{\mathrm{SP}}=-0,01 \cdot \mathrm{U}_{\mathrm{z}}^{2}+51,4 \cdot \mathrm{U}_{\mathrm{z}}+28603$ & $\mathrm{R}^{2}=0,99$ \\
\hline & $\mathrm{W}_{\mathrm{SP}}=-6,9 \cdot t_{d w e l l}{ }^{2}+60,2 \cdot t_{\text {dwell }}-38,1$ & $\mathrm{R}^{2}=0,99$ \\
\hline & $\mathrm{W}_{\mathrm{SP}}=0,2 \cdot \mathrm{T}^{2}+13,1 \cdot \mathrm{T}-77,1$ & $\mathrm{R}^{2}=0,99$ \\
\hline \multirow{3}{*}{$\begin{array}{c}\text { Duração da } \\
\text { Faísca (tsP) }[\mathrm{ms}]\end{array}$} & $\mathrm{t}_{\mathrm{SP}}=1 \cdot 10^{-6} \cdot \mathrm{U}_{\mathrm{z}}{ }^{2}-0,004 \cdot \mathrm{U}_{\mathrm{z}}+4,3$ & $\mathrm{R}^{2}=0,99$ \\
\hline & $\mathrm{t}_{\mathrm{SP}}=-0,1 \cdot t_{\text {dwell }}{ }^{2}+0,8 \cdot t_{\text {dwell }}+0,3$ & $\mathrm{R}^{2}=0,99$ \\
\hline & $\mathrm{t}_{\mathrm{SP}}=0,1 \cdot \mathrm{T}+0,4$ & $\mathrm{R}^{2}=0,96$ \\
\hline
\end{tabular}

OBS.: Tensão de grampeamento $\left(\mathrm{U}_{\mathrm{z}}\right)$ em $\mathrm{V}$, Tensão de entrada $(\mathrm{T})$ em $\mathrm{V}$ e dwell time $\mathrm{t}_{\mathrm{dwell}}$ em ms.

Assim como no ensaio de oferta, todas as equações obtidas por meio do software Microsoft Excel® obtiveram um bom coeficiente de discriminação $\left(\mathrm{R}^{2} \geq 0,96\right)$, ou seja, as equações representam bem o comportamento observado em bancada. Cabe ressaltar que, tais equações são válidas apenas para a faixa de entrada das grandezas abordadas neste estudo, para outros valores o comportamento da bobina pode ser diferente do observado neste trabalho. 


\section{CONCLUSÃO}

Os resultados observados neste estudo demonstraram que variações nas grandezas de entrada podem vir a comprometer a confiabilidade dos resultados obtidos nos ensaios funcionais de Oferta de Tensão e Energia. Logo, este trabalho serve de base para um melhor entendimento do comportamento do sistema de medição, além de possibilitar identificar as possíveis fontes de variação, quando obtidos resultados inesperados.

Além disso, todas as equações calculadas por meio das regressões apresentaram um bom coeficiente de discriminação, sempre acima de 96\%, ou seja, representam, de forma aceitável, o comportamento observado em bancada. Desta forma, por meio das equações obtidas, os coeficientes de sensibilidades, utilizados para expressar a incerteza de medição, podem ser adquiridos, facilitando futuros cálculos.

Por fim, conclui-se que, com o estudo de causa e efeito das grandezas de entrada e saída dos ensaios funcionais de Oferta de Tensão e Energia, os usuários de tais sistemas de medição dispõem de uma base de dados nos quais podem ser utilizados para estudos futuros. Ademais, possíveis pontos de melhoria no sistema de medição poderão ser identificados, podendo, dessa forma, promover o desenvolvimento de seus sistemas, por meio de equipamentos mais precisos e exatos.

\section{AGRADECIMENTOS}

Este trabalho foi realizado durante uma parceria entre o Programa INOVA Talentos, fomentado pelo Ministério da Ciência, Tecnologia, Inovações e Comunicações (MCTIC), Conselho Nacional de Desenvolvimento Científico e Tecnológico (CNPq), Instituto Euvaldo Lodi (IEL) e a empresa Robert Bosch Ltda.

Os autores agradecem a empresa Robert Bosch Ltda. por todo incentivo e pela disponibilidade de recursos para a realização deste trabalho.

\section{REFERÊNCIAS}

1. Ignition Systems for Gasoline Engines. 1st edition. Alemanha: Robert Bosch GmbH, 2003.

2. CAMPI, Marcelo Sartori. Fatores de influência para otimização do nível de emissão irradiada do sistema de ignição. Anais do XXIII Simpósio Internacional de Engenharia Automotiva - SIMEA 2014. p. 222-228. São Paulo: Blucher, 2015.

3. MILHOR, Carlos Eduardo. Sistema de desenvolvimento para controle eletrônico dos motores de combustão interna ciclo otto. Dissertação de Mestrado. Escola de Engenharia de São Carlos; Universidade de São Paulo; São Carlos; 2002.

4. Lei da Indução de Faraday. Apostila. Laboratório de Eletricidade e Magnetismo. Instituto de Física de São Carlos, Universidade de São Paulo, São Paulo.

5. Bosch Ignition System Training. Alemanha: Robert Bosch GmbH, 2012.

6. Test Specification ZSK 1x1 Brasil: Robert Bosch Ltda. (Intern Document) 